\title{
Paired box 2 induces epithelial-mesenchymal transition in normal renal tubular epithelial cells of rats
}

\author{
$\mathrm{LI} \mathrm{LI}^{1,2}$, YUBIN WU ${ }^{1}$ and YONGCHANG YANG ${ }^{1}$ \\ ${ }^{1}$ Department of Pediatric Nephrology and Rheumatology, Shengjing Hospital of China Medical University, \\ Shenyang, Liaoning 110004; ${ }^{2}$ Department of Pediatrics, The First Affiliated Hospital \\ of Liaoning Medical College, Jinzhou, Liaoning 121001, P.R. China
}

Received November 13, 2012; Accepted January 29, 2013

DOI: $10.3892 / \mathrm{mmr} .2013 .1365$

\begin{abstract}
Epithelial-mesenchymal transition (EMT) is considered to be a crucial stage of renal fibrosis. Previous studies have indicated that paired box 2 (PAX2) affects renal fibrosis, likely by regulating EMT. Therefore, we examined whether PAX2 directly induces normal renal tubular EMT in vitro. Recombinant PAX2 plasmid was transfected into a renal tubular epithelial cell line (NRK52E) derived from normal rats. The optimal time-point was evaluated through fluorescence detection, western blotting and real-time polymerase chain reaction (PCR). Morphological alterations were examined using phase-contrast microscopy. At the optimal time-point of transfection, the expression of E-cadherin, $\alpha$-smooth muscle actin ( $\alpha$-SMA), fibronectin and snail was analyzed by western blotting and real-time PCR. PAX2 expression in NRK52E cells transfected with PAX2 plasmid reached its peak at $72 \mathrm{~h}$ post-transfection. The transfected cells became elongated. PAX2 transfection induced a fibrotic phenotype of NRK52E cells with increased expression of $\alpha$-SMA, fibronectin and snail as well as decreased expression of E-cadherin. The optimal time-point for transfection efficiency was $72 \mathrm{~h}$. In conclusion, PAX2 induces renal tubular mesenchymal transition in vitro.
\end{abstract}

\section{Introduction}

Renal fibrosis, characterized by tubulointerstitial fibrosis and glomerulosclerosis, is the final manifestation of chronic kidney disease (CKD). With the progression of CKD, patients inevitably reach endstage renal disease and require renal replacement therapies, such as dialysis and transplantation. Renal fibrosis is the final result of CKD. Transformation in the phenotype of the cell epithelial-mesenchymal transition (EMT) is considered

Correspondence to: Professor Yubin Wu, Department of Pediatric Nephrology and Rheumatology, Shengjing Hospital of China Medical University, No. 36 Shanhao Street, Heping, Shenyang, Liaoning 110004, P.R. China

E-mail: yubinwucn@126.com

Key words: epithelial-mesenchymal transition, gene transfer, paired box 2, NRK52E to greatly contribute to renal fibrosis. Epithelial cells that have undergone EMT are characterized by the loss of epithelial cell markers, such as E-cadherin, and de novo mesenchymal cell markers, such as $\alpha$-smooth muscle actin ( $\alpha$-SMA).

EMT, as an important physiological event, occurs frequently during embryonic development (1). However, numerous studies have demonstrated that EMT also occurs during pathological conditions such as tumor metastasis and tissue fibrosis (2-4). Various studies have confirmed that EMT plays a crucial role in the development and progression of renal fibrosis (5).

Paired box 2 (PAX2) gene encodes a nuclear transcription factor which is key to inducing the renal tubular epithelial cell transition during embryonic development. This plays an important role in regulating the embryonic development of the kidney at various stages (6). However, in the perinatal period, new nephron formation is hindered when the expression of PAX2 is sharply suppressed. Aberrant PAX2 expression in renal tubular epithelial cells has been reported in children with glomerular diseases, suggesting that restoration of PAX2 may result in renal tubular epithelial cell transdifferentiation (7). We have previously observed that in a unilateral ureteral obstruction (UUO) model, the reactivation of PAX2 occurs primarily in the renal tubular epithelial cells, suggesting its role in regulating EMT (8).

However, the effect of PAX2 on EMT has not yet been elucidated. In the present study, we investigated whether PAX2 induces EMT in NRK52E cells in vitro in order to provide experimental evidence for the potential role of PAX2 in the prevention and treatment of renal interstitial fibrosis.

\section{Materials and methods}

Animals and UUO model. Four-week-old male Wistar rats (weighing 120-150 g) were obtained from the Shengjing Hospital Laboratory Animal Center (Shenyang, China). This study was performed in strict accordance with the recommendations in the Guide for the Care and Use of Laboratory Animals of the National Institutes of Health. The animal use protocol was reviewed and approved by the Institutional Animal Care and Use Committee of Shengjing Hospital of China Medical University. The rats were anesthetized via intraperitoneal injection of $10 \%$ chloral hydrate (Shengjing Hospital, Shenyang, China). The left ureter was exposed and 
Table I. Real-time RT-PCR primers used in the study and amplification product lengths.

\begin{tabular}{llc}
\hline Target & \multicolumn{1}{c}{ Sequence $\left(5^{\prime} \rightarrow 3^{\prime}\right)$} & Product length $(\mathrm{bp})$ \\
\hline$\beta$-actin & F: ACGTTGACATCCGTAAAGAC & 200 \\
& R: GAAGGTGGACAGTGAGGC & 195 \\
PAX2 & F: CAACGGTGAGAAGAGGAAACGAG & \\
& R: TAATGCTGCTGGGTGAAGGTGTC & 211 \\
E-SMA & F: CTCATCCACGAAACCACCTAT \\
& R: CGCCGATCCAGACAGAATA & 172 \\
& F: AAAGCAGGAAGAAAACACCACTC & \\
& R: AAAGGGCACGCTATCAACATTAG &
\end{tabular}

RT-PCR, reverse transcription-polymerase chain reaction; PAX2, paired box 2; $\alpha$-SMA, $\alpha$-smooth muscle actin; F, forward; R, reverse.

ligated with 3-0 silk through a midline abdominal incision. The rats were sacrificed 21 days following UUO.

Complementary DNA (cDNA) synthesis. Total RNA was extracted from the renal cortex of the rats using TRIzol reagent (Invitrogen, Carlsbad, CA, USA) according to the manufacturer's instructions. Total RNA (500 ng) was reverse-transcribed into cDNA using the reverse transcription (RT) reagent kit according to the manufacturer's guidelines. The full-length PAX2 gene was amplified by polymerase chain reaction (PCR) from PAX2 cDNA, using the following primers: forward, GAGGATCCCCGGGTACCGGTCGCCACCATGGATATG CACTGCAAAG and reverse, CACCATGGTGGCGACCGG GTGGCGGTCATAGGCAGC containing AgeI sites. The target sequence was amplified by PCR at $94^{\circ} \mathrm{C}$ for $5 \mathrm{~min}, 94^{\circ} \mathrm{C}$ for $30 \mathrm{sec}, 55^{\circ} \mathrm{C}$ for $30 \mathrm{sec}, 72^{\circ} \mathrm{C}$ for $1.5 \mathrm{~min}$ for 30 cycles, and $72^{\circ} \mathrm{C}$ for $10 \mathrm{~min}$.

Construction of $p G C-F U-G F P-P A X 2$ plasmid. Following $\mathrm{PCR}$, the PCR products of the PAX2 gene were purified to generate the pGC-FU-GFP transfer vector (GeneChem Co., Ltd., Shanghai, China). The ligated products were transformed into TOP10 chemically competent Escherichia coli (Takara, Otsu, Shiga, Japan) and incubated on Luria-Bertani plates containing $100 \mu \mathrm{g} / \mathrm{ml}$ ampicillin at $37^{\circ} \mathrm{C}$ overnight. Subsequently, 8 putative ampicillin-resistant positive clones were selected to amplify, extract and purify for PCR amplification and electrophoresis detection. The sequencing was completed by Shanghai ShengGong Biotechnology Co., Ltd. (Shanghai, China).

Cell cultures and transient transfection. The well-characterized normal rat renal tubular epithelial cell line NRK52E was obtained from the American Type Culture Collection (Rockville, MD, USA). The NRK52E cells were cultured in Dulbecco's modified Eagle's medium (Invitrogen) with $10 \%$ fetal calf serum and $1 \%$ penicillin/streptomycin solution $(\mathrm{P} / \mathrm{S}$; cat. no. 0503 ) at $37^{\circ} \mathrm{C}$ in a $5 \% \mathrm{CO}_{2}$ atmosphere. Cells were plated onto 6-well plates at a density of $2 \times 10^{5}$ cells/well, grown overnight, and then transferred to serum-free medium prior to PAX2 transfection. NRK52E cells were transfected with $4 \mu \mathrm{g}$ pGC-FU-GFP-PAX2 plasmids or empty vector using

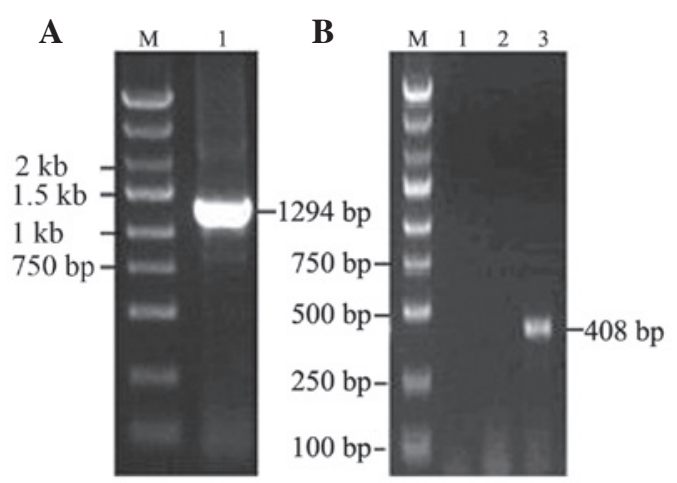

Figure 1. Construction of pGC-FU-GFP vector. (A) Rat PAX2 gene was amplified using PCR. Lane M, DNA ladder; lane 1, PCR product (1,294 bp). (B) Identification of the PCR products of positive clones of plasmid. Lane M, DNA ladder; lane 1, dd $\mathrm{H}_{2} \mathrm{O}$; lane 2, empty pGC-FU-GFP vector; lane 3, PAX2 translator. PAX2, paired box 2; PCR, polymerase chain reaction.

$5 \mu$ Lipofectamine LM $^{\mathrm{TM}} 2000$ transfection reagent (Invitrogen) according to the manufacturer's instructions. Cells were observed for green fluorescent protein (GFP) using fluorescence microscopy after 24, 48, 72 and $96 \mathrm{~h}$ transfection. The control cells were grown in serum-free medium alone. All the experiments were performed in triplicate.

Phase contrast microscopy. After phase contrast microscopy was performed using a charge-coupled device (CCD) video camera attached to a TMS phase contrast microscope (Nikon, Tokyo, Japan), all the micrographs were subsequently processed using Adobe Photoshop software.

Western blotting. Protein concentrations were determined using a BCA protein assay kit (Sigma-Aldrich, Seelze, Germany). Samples were heated at $100^{\circ} \mathrm{C}$ for 5-10 min before loading and were separated on precast 10 or $5 \%$ SDS-polyacrylamide gels (Bio-Rad, Hercules, CA, USA). The proteins were electrotransferred onto nitrocellulose membranes (Amersham, Arlington Heights, IL, USA) in transfer buffer. Following transfer, all incubations were conducted on a rocking platform at room temperature. The membranes were blocked in 5\% skimmed milk/TBST overnight, then incubated for $1 \mathrm{~h}$ with PAX2 (1:700; Zymed, Carlsbad, CA, USA), E-cadherin, fibronectin, snail 


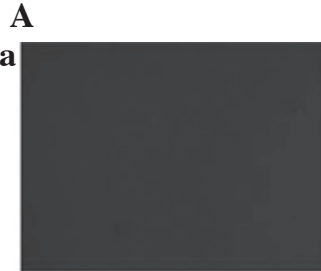

Control

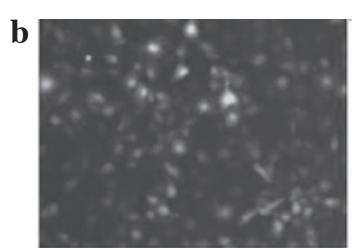

Empty vector

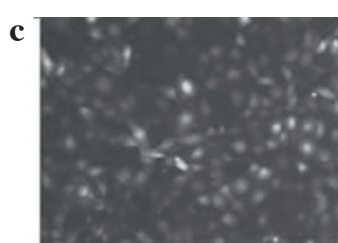

$48 \mathrm{~h}$

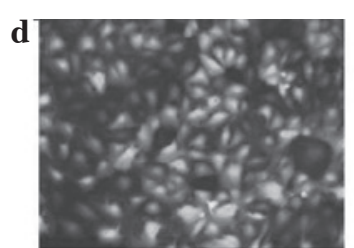

$72 \mathrm{~h}$

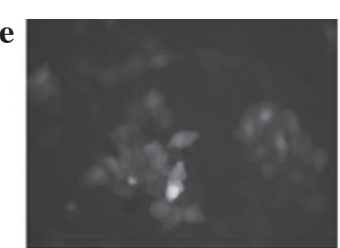

$96 \mathrm{~h}$
B
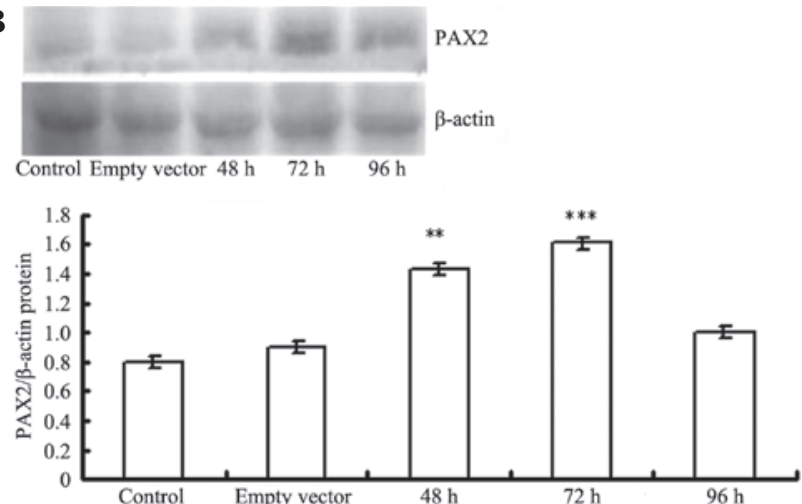

$\mathbf{C}$

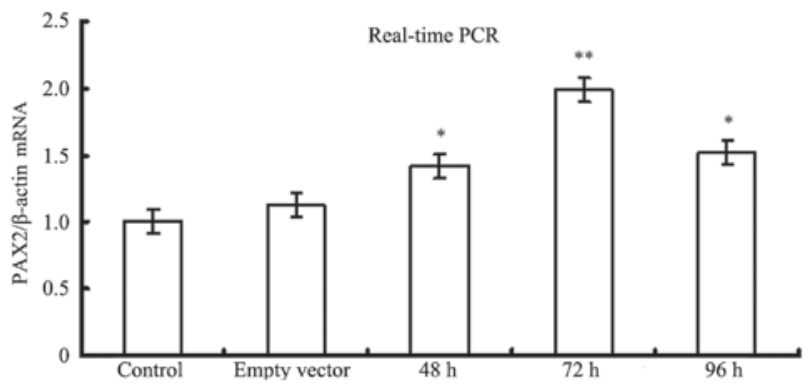

Figure 2. Identification of pGC-FU-GFP-PAX2 plasmid. (A) Expression of green fluorescent protein in NRK52E cells was detected by fluorescence microscopy (magnification, x200) after NRK52E cells were transfected with pGC-FU-GFP-PAX2 plasmid. (B) Western blotting and (C) real-time PCR analysis of PAX2 protein and mRNA in NRK52E cells transfected with and without plasmid (control). The results shown are expressed as the ratio of PAX2 to $\beta$-actin. ${ }^{* *} \mathrm{P}<0.01$ (protein) at $48 \mathrm{~h}$ and ${ }^{*} \mathrm{P}<0.05$ (mRNA) at $48 \mathrm{~h}, \mathrm{PAX} 2$ vs. control; ${ }^{* * *} \mathrm{P}<0.001$ (protein) and ${ }^{* *} \mathrm{P}<0.01$ (mRNA), when maximum expression was reached at $72 \mathrm{~h}$. PAX2, paired box 2 ; PCR, polymerase chain reaction.

(1:1,000; Santa Cruz Biotechnology, Inc., Santa Cruz, CA, USA) and $\alpha$-SMA (1:1,500; Sigma, St. Louis, MO, USA) primary antibodies. The membranes were washed with Tris-buffered saline (TBS) and incubated with a goat anti-mouse horseradish peroxidase-conjugated secondary antibody for $1 \mathrm{~h}$. Membranes were then washed with TBS buffer, and the signals were visualized using the enhanced chemiluminescence system (Amersham).

Real-time PCR. Total RNA was extracted from the cells. cDNA was synthesized using 500 ng total RNA with the PrimeScript ${ }^{\mathrm{TM}}$ RT-PCR kit (Takara). Real-time PCR was performed using the SYBR-Green real-time PCR Master mix (Takara). Primers of PAX2, E-cadherin, $\alpha$-SMA, fibronectin, snail and the internal control $\beta$-actin were synthesized by Invitrogen (Table I). Reactions were amplified in a Roche LightCycler ${ }^{\circledR}$ (Mannheim, Germany) under the following conditions: initial denaturation at $95^{\circ} \mathrm{C}$ for $5 \mathrm{~min}, 35$ cycles of denaturation at $95^{\circ} \mathrm{C}$ for $20 \mathrm{sec}$, annealing at $60^{\circ} \mathrm{C}$ for $20 \mathrm{sec}$, and polymerization at $72^{\circ} \mathrm{C}$ for $30 \mathrm{sec}$. PCR products were analyzed by agarose electrophoresis. Standard curves were established using serial dilutions of sample cDNA. These curves were used to measure the expression levels of the target gene and the reference $\beta$-actin gene using Roche LightCycler ${ }^{\circledR}$ software 4.05. Expression of the target gene was normalized to $\beta$-actin expression.

Statistical analysis. SPSS13.0 software was used for statistical analysis. The differences between the groups were assessed using Student's t-test. $\mathrm{P}<0.05$ was considered to indicate a statistically significant difference. The following scheme was used throughout the results: ${ }^{*} \mathrm{P}<0.05,{ }^{* *} \mathrm{P}<0.01$ and ${ }^{* * *} \mathrm{P}<0.001$. Cells from three wells were analyzed for each experiment, which was performed at least thrice.

\section{Results}

Construction and identification of $p G C-F U-G F P-P A X 2$ plasmid. PAX2 mRNA was extracted from the kidney of a UUO model rat. After PAX2 was digested by AgeI, a 1,294-bp fragment of PAX2 was directly cloned into the pGC-FU-GFP vector. Approximately 408-bp long DNA bands were observed in those positive clones (Fig. 1). The positive clones were sent to Shanghai Invitrogen Biotechnology Co., Ltd. After using BLAST in GenBank, PAX2 sequences were successfully cloned into the eukaryotic expression vector pGC-FU-GFP.

Identification of $p G C-F U-G F P-P A X 2$ plasmid transfection. Fluorescence microscopy analysis showed that at $72 \mathrm{~h}$ post-transfection of pGC-FU-GFP-PAX2, the intensity of GFP became markedly stronger compared with that of controls and other time-points. Expression of PAX2 protein began to increase $48 \mathrm{~h}$ post-transfection. The expression level peaked at $72 \mathrm{~h}$, and then decreased at $96 \mathrm{~h}$. Real-time PCR showed the same result (Fig. 2). These results indicate that $72 \mathrm{~h}$ is the optimal time-point during PAX2 transfection.

PAX2 induces mesenchymal morphology of NRK52E cells. Control and empty vector NRK52E cells showed a typical epithelial cuboidal shape, with cobblestone morphology. Transfection with PAX2 caused distinct morphological changes, with evidence of gross elongation, after $72 \mathrm{~h}$ of transfecting PAX2 in NRK52E cells (Fig. 3).

$P A X 2$ reduces the expression of E-cadherin in tubular epithelial cells. E-cadherin is a tubular epithelial cell-cell adhesion receptor that is essential for the formation and maintenance 

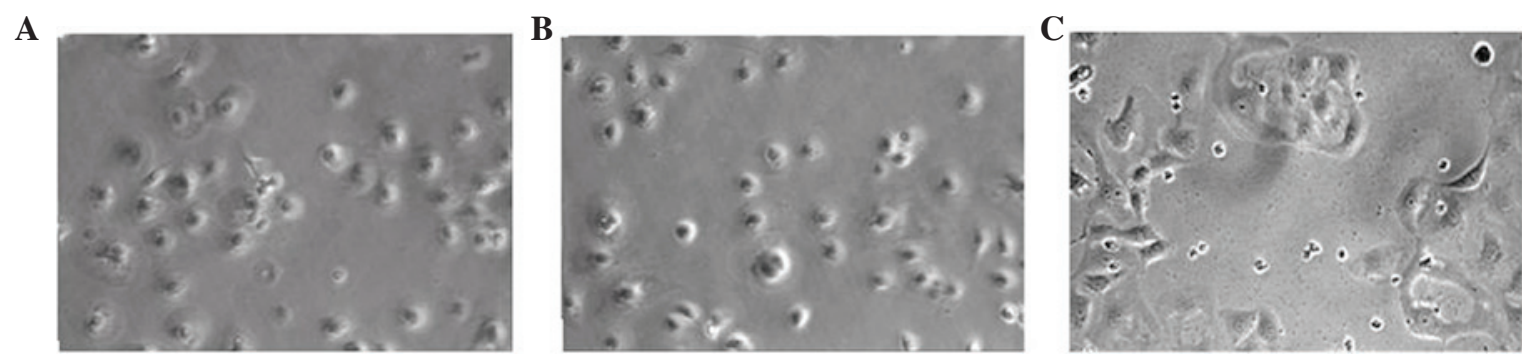

Figure 3. Effect of PAX2 transfection on NRK52E cellular morphology. NRK52E cells were transfected (A) without (control), (B) with empty vector and (C) with PAX2 for $72 \mathrm{~h}$ to induce epithelial-mesenchymal transition. Cells were observed using phase contrast microscopy. The images are representative of at least 3 independent experiments performed in duplicate (magnification, x100). PAX2, paired box 2.
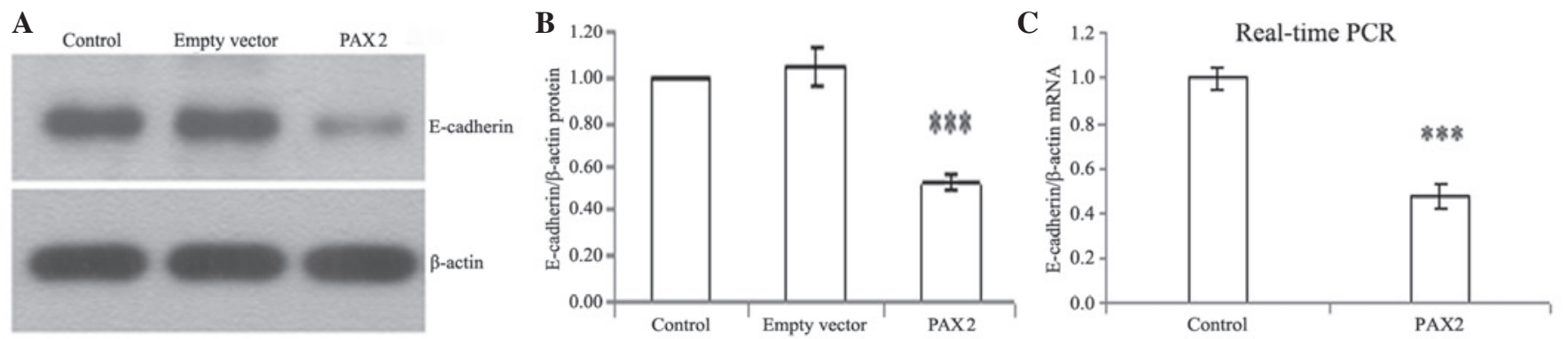

Figure 4. E-cadherin expression in tubular epithelial cells. (A and B) Western blotting and (C) real-time PCR of E-cadherin protein and mRNA expression in NRK52E cells transfected with or without PAX2 (control). The graph summarizes densitometric analysis of 3 independent experiments. The results shown are expressed as the ratio of E-cadherin to $\beta$-actin; ${ }^{* * *} \mathrm{P}<0.001$, PAX2 vs. control. PAX2, paired box 2; PCR, polymerase chain reaction.

of the homeostasis and architecture of renal epithelia. It was observed that PAX2 markedly repressed E-cadherin expression in NRK52E cells. Western blotting and real-time PCR revealed significant suppression of E-cadherin protein and mRNA expression levels following PAX2 transfection for $72 \mathrm{~h}$ (Fig. 4).

PAX2 induces the expression of $\alpha$-SMA, fibronectin and snail. To determine whether tubular epithelial cells undergo conversion into myofibroblasts in vitro, we used NRK52E cells as a model system to examine the ability of PAX2 to induce the de novo expression of $\alpha$-SMA, fibronectin and snail, which are the phenotypic markers of myofibroblasts. PAX2 markedly induced protein and mRNA expression of $\alpha$-SMA, fibronectin and snail in NRK52E cells (Figs. 5-7).

\section{Discussion}

PAX2 is a member of the paired box class of nuclear transcription factors, and it plays a pivotal role during the early stages of renal epithelial differentiation. During kidney development, PAX2 is present in the caudal mesonephric duct, the ureteric bud, and at a later stage, in the mesenchymal condensates that are induced by the bud (9). As the mesenchymal condensates convert into nephron epithelia, PAX2 expression is repressed. Its suppression is essential for the terminal differentiation of mesenchymal-epithelial transformation. The increase in PAX2 in transgenic mice leads to severe kidney abnormalities (10), and PAX2-null mice lack kidneys, ureters and genital tracts (11). Over- or underexpression of PAX2 in humans results in renal diseases. Murer et al (12) showed that PAX2 is overexpressed in juvenile nephronophthisis. It was also observed that PAX2 was expressed in immature dysplastic tubules, and that it was repressed in mature renal tubular cells. In addition, PAX2 was present in severe secondary interstitial fibrosis. The failure of PAX2 repression or the reactivation of PAX2 in juvenile nephronophthisis probably leads to a primary defect along the cascade of mesenchymal epithelial differentiation and may be involved in interstitial fibrosis and cyst formation. Huang et al (13) observed the re-expression of PAX2 in 5/6 nephrectomized rats. These results indicated that the re-expression of PAX2 may be significant in the process of EMT. Similarly to those results, in 2010, we showed that PAX2 was re-expressed in the renal tubular epithelium in a rat UUO obstructive kidney model (8). PAX2 expression may have resulted from the initiation of renal fibrosis regulatory mechanisms, and then activated the renal tubular epithelial cell transdifferentiation. However, to date, PAX2 has not been shown to be directly involved in renal tubular EMT.

During EMT, the following four key events are crucial (14-17): loss of epithelial adhesion properties, such as in E-cadherin; acquired expression of $\alpha$-SMA reorganization; disruption of the tubular basement membrane; and enhanced cell migration and invasion. Our studies showed that PAX2 is capable of inducing tubular epithelial cells to undergo transferation, which involves three of these events. Although we did not study cell motility and migration, the PAX2-induced morphologic alterations, which included the elongation observed using phase contrast microscopy, are consistent with the improved migratory capability of the transformed cells. We observed that the expression of the epithelial cell adhesion 
A

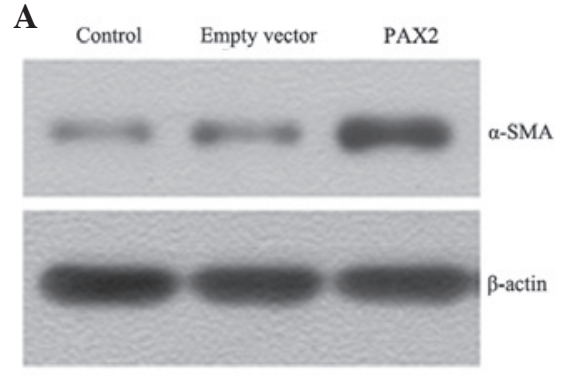

B

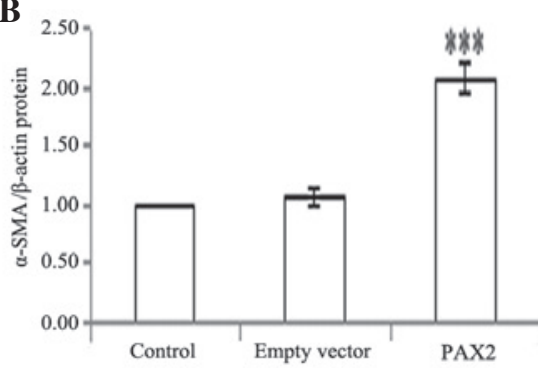

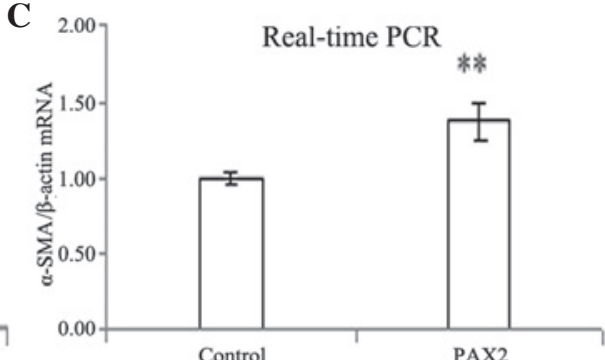

Figure 5. $\alpha$-SMA expression in tubular epithelial cells. (A and B) Western blotting and (C) real-time PCR of $\alpha$-SMA protein and mRNA expression in NRK52E cells transfected with or without PAX2 (control). The graph summarizes densitometric analysis of 3 independent experiments. The results shown are expressed as the ratio of $\alpha$-SMA to $\beta$-actin; ${ }^{* * * *} \mathrm{P}<0.001$, PAX2 vs. control protein; ${ }^{* *} \mathrm{P}<0.01, \mathrm{PAX} 2$ vs. control mRNA. $\alpha$-SMA, $\alpha$-smooth muscle actin; PAX2, paired box 2; PCR, polymerase chain reaction.
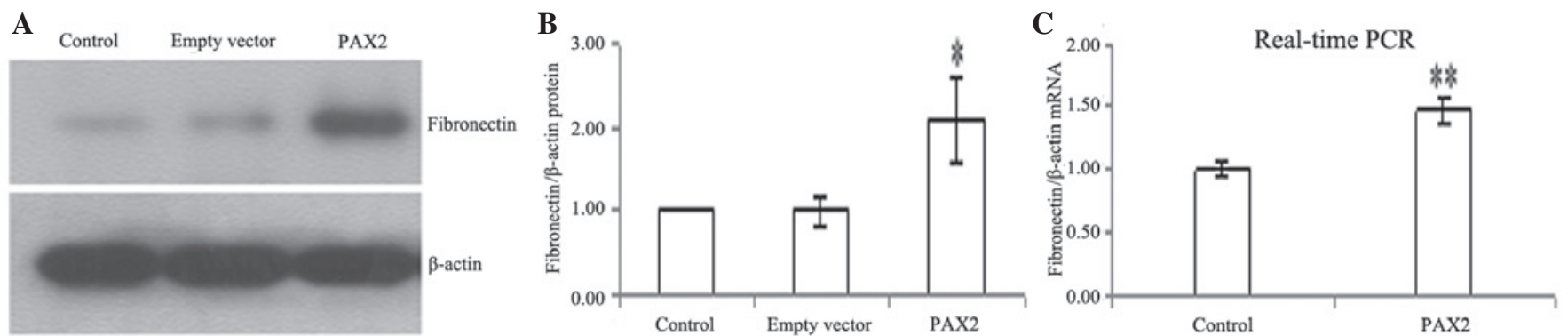

Figure 6. Fibronectin expression in tubular epithelial cells. (A and B) Western blotting and (C) real-time PCR of fibronectin protein and mRNA expression in NRK52E cells transfected with or without PAX2 (control). The graph summarizes densitometric analysis of 3 independent experiments. The results shown are expressed as the ratio of fibronectin to $\beta$-actin; ${ }^{* * *} \mathrm{P}<0.05$, PAX2 vs. control protein; ${ }^{* *} \mathrm{P}<0.01$, PAX2 vs. control mRNA. PAX2, paired box 2 ; PCR, polymerase chain reaction.
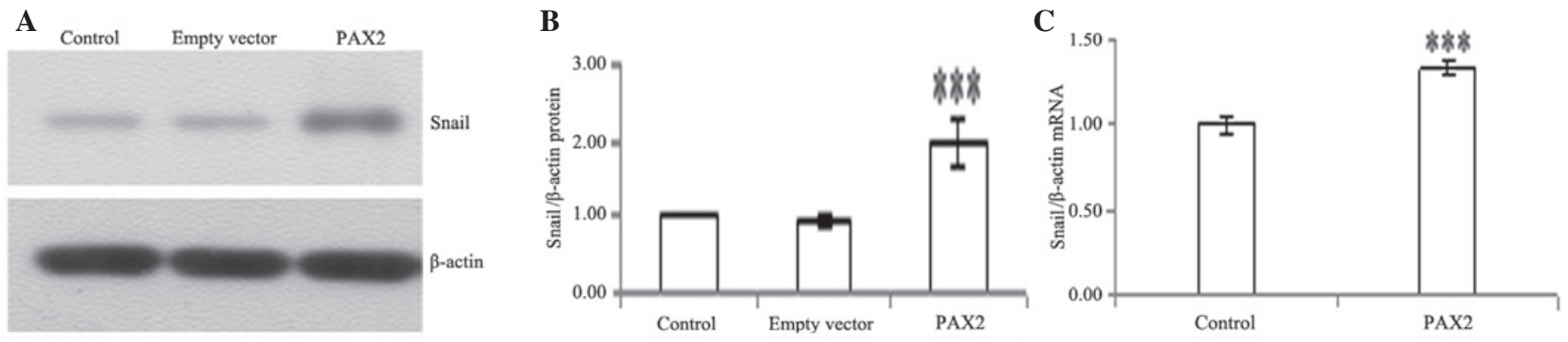

Figure 7. Snail expression in tubular epithelial cells. (A and B) Western blotting and (C) real-time PCR of snail protein and mRNA expression in NRK52E cells transfected with or without PAX2 (control). The graph summarizes densitometric analysis of 3 independent experiments. The results shown are expressed as the ratio of snail to $\beta$-actin; ${ }^{* * *} \mathrm{P}<0.001$, PAX2 vs. control. PAX2, paired box 2 ; PCR, polymerase chain reaction.

molecule E-cadherin decreased $72 \mathrm{~h}$ post-transfection, whereas the expression of $\alpha$-SMA, fibronectin and snail mesenchymal markers increased in NRK52E cells $72 \mathrm{~h}$ post-transfection, indicating that these cells lost their epithelial characteristics and acquired mesenchymal cell properties. These results showed that PAX2 induces the phenotypic and morphologic alterations of renal tubular epithelial cells in vitro.

Doberstein et al (18) reported that PAX2 directly binds to ADAM10 promoter and regulates the expression of ADAM10. Moreover, PAX2 has been shown to be a regulator of L1-CAM expression. The downregulation of PAX2 may lead to EMT of renal cancer cells by downregulation of ADAM10 or the induction of release of soluble L1-CAM. These results appear to be contradictory to the findings of the present study. A potential explanation for this result is that the studies were carried out in different cells. The different transdifferentiation effects of PAX2 may be due to the different intracellular environments of the renal carcinoma cells and normal renal tubular epithelial cells of rats.

In our previous study, it was demonstrated that PAX2 was re-expressed in rats with obstructive nephropathy and may participate in the pathogenesis of renal tubular damage and renal interstitial fibrosis (8). Huang et al (8) showed that silencing of PAX2 by RNA interference blocked the interleukin-1-induced EMT in NRK52E cells, as reflected in the suppression of $\alpha$-SMA, the restoration of E-cadherin expression and normal cell morphology (19). Thus, it is reasonable to speculate that re-expression of PAX2 activates the renal tubular 
epithelial cell transdifferentiation procedure and promotes the renal fibrosis process. Blocking PAX2 may reverse EMT and prevent or alleviate renal fibrosis. Zhou et al (20) demonstrated that prohibitin and PAX2 are associated with the development of renal interstitial fibrosis. Decreased expression of prohibitin is associated with increased PAX2 gene expression and renal interstitial fibrosis index in UUO rats. In conclusion, the mechanism by which PAX2 induces EMT requires further investigation.

\section{Acknowledgements}

This study was supported by grants from the Scientific Technique Project of Shengyang (F10-205-1-29) and the Doctor Excellent Project Fund of Shengjing Hospital of China Medical University (ma26).

\section{References}

1. Potenta S, Zeisberg E and Kalluri R: The role of endothelial-tomesenchymal transition in cancer progression. Br J Cancer 99: 1375-1379, 2008.

2. Strutz F, Okada H,Lo CW, et al: Identification and characterization of a fibroblast marker: FSP1. J Cell Biol 130: 393-405, 1995.

3. Zeisberg M, Yang C, Martino M, et al: Fibroblasts derive from hepatocytes in liver fibrosis via epithelial to mesenchymal transition. J Biol Chem 282: 23337-23347, 2007.

4. Kim KK, Kugler MC, Wolters PJ, et al: Alveolar epithelial cell mesenchymal transition develops in vivo during pulmonary fibrosis and is regulated by the extracellular matrix. Proc Nat Acad Sci USA 103: 13180-13185, 2006.

5. Zeisberg EM, Potenta SE, Sugimoto H, Zeisberg M and Kalluri R: Fibroblasts in kidney fibrosis emerge via endothelial-to-mesenchymal transition. J Am Soc Nephrol 19: 2282-2287, 2008.

6. Narlis M, Grote D, Gaitan Y, Boualia SK and Bouchard M: PAX2 and Pax8 regulate branching morphogenesis and nephron differentiation in the developing kidney. J Am Soc Nephrol 18: 1121-1129, 2007.

7. Geng WM, Yi ZW, He XJ, et al: Function of PAX2 in tubular epithelium transdifferentiation. J Clin Pediatr 25: 284-287, 2007 (In Chinese).
8. Li L, Wu YB and Zhang WG: PAX2 re-expression in renal tubular epithelial cells and correlation with renal interstitial fibrosis of rats with obstructive nephropathy. Ren Fail 32: 603-611, 2010.

9. Eccles MR, He S, Legge M, et al: PAX genes in development and disease: the role of PAX2 in urogenital tract development. Int J Dev Biol 46: 535-544, 2002.

10. Torres M, Gómez-Pardo E, Dressler GR and Gruss P: Pax-2 controls multiple steps of urogenital development. Development 121: 4057-4065, 1995.

11. Dziarmaga A, Eccles M and Goodyer P: Suppression of ureteric bud apoptosis rescues nephron endowment and adult renal function in Pax2 mutant mice. J Am Soc Nephrol 17: 1568-1575, 2006.

12. Murer L, Caridi G, Della Vella M, et al: Expression of nuclear transcription factor PAX2 in renal biopsies of juvenile nephronophthisis. Nephron 91: 588-593, 2002.

13. Huang B, Pi L, Chen C, et al: WT1 and Pax 2 re-expression is required for epithelial-mesenchymal transition in 5/6 nephrectomized rats and cultured kidney tubular epithelial cells. Cells Tissues Organs 195: 296-312, 2012.

14. Rastaldi MP: Epithelial-mesenchymal transition and its implications for the development of renal tubulointerstitial fibrosis. J Nephrol 19: 407-412, 2006.

15. Liu Y: Epithelial to mesenchymal transition in renal fibrogenesis: pathologic significance, molecular mechanism, and therapeutic intervention. J Am Soc Nephrol 15: 1-12, 2004.

16. Rastaldi MP,FerrarioF, Giardino L, et al: Epithelial-mesenchymal transition of tubular epithelial cells in human renal biopsies. Kidney Int 62: 137-146, 2002.

17. Yang J and Liu Y: Dissection of key events in tubular epithelial to myofibroblast transition and its implications in renal interstitial fibrosis. Am J Pathol 159: 146-1475, 2001.

18. Doberstein K, Pfeilschifter J and Gutwein P: The transcription factor PAX2 regulates ADAM10 expression in renal cell carcinoma. Carcinogenesis 32: 1713-1723, 2011.

19. Doberstein K, Wieland A, Lee SB, et al: L1-CAM expression in ccRCC correlates with shorter patients survival times and confers chemoresistance in renal cell carcinoma cells. Carcinogenesis 32: 262-270, 2011.

20. Zhou TB, Zeng ZY, Qin YH and Zhao YJ: Less expression of prohibitin is associated with increased paired box 2 (PAX2) in renal interstitial fibrosis rats. Int J Mol Sci 13: 9808-9825, 2012. 